\title{
When will On-Orbit Servicing be part of the space enterprise?
}

\author{
Daniel E. Hastings ${ }^{1}$, Benjamin L. Putbrese \& Paul A. La Tour
}

Engineering Systems Division, MIT, Cambridge, MA 02139

\begin{abstract}
The space industry is currently at a significant inflection point. Over the past decades, many spacecraft at geosynchronous orbit have continued a trend towards increasingly massive and longer-lasting satellites, and while they do represent some of the most exquisite, highest-performing satellites ever launched, some experts now feel that such trends are unsustainable and are beginning to place increasing strain on the underlying industry. To support current and future spacecraft, on-orbit servicing (OOS) infrastructures have been proposed, which would provide services such as repair, rescue, refueling, and upgrading of customer spacecraft in order to alleviate the identified space industry trends. In this paper, system dynamics modeling is used to assess various scenarios for OOS incorporation into the overall space industry, by evaluating its long-term effects on the design, cost, and underlying experience of a reference geosynchronous constellation. This system dynamics model is based heavily in behavioral economics' Prospect Theory, with such concepts as anchoring and loss aversion factoring heavily into the overall simulation of the space industry. The primary conclusion of this analysis was that relatively low costs and substantial incorporation of servicing capabilities into customer architectures are likely to be necessary to ensure long-term sustainability of such a project. Finally, several policy implications for an OOS infrastructure are outlined.
\end{abstract}

\section{Introduction}

The ability to repair, refuel, and reposition spacecraft in orbit has often been proposed as a means of radically restructuring the conduct and planning of space missions. As the predominant spacecraft design paradigm stands today, once a satellite has been placed into its predetermined orbit, there is absolutely no way for engineers to in any way modify or fix that satellite, beyond changes in software or selfrepositioning (which is likely impossible, or at least very undesirable given the large amount of precious on-board fuel which is required for significant orbital maneuvers). Naturally, this paradigm places significant constraints on satellite design parameters and mission flexibility. Also, should an anomaly strike, mechanical or otherwise, before the end of a satellite's operational lifetime, then options for repair and recovery of prior levels of service are very limited. While engineers have certainly devised ingenious methods for salvaging value from damaged or malfunctioning spacecraft in the past (for instance, by shifting a mission's concept of operations to accommodate the spacecraft as is), few would dismiss a means of fixing (or upgrading) the spacecraft and continuing its mission as originally planned, if such a service was available.

This paper explores in the overall space industry response to the implementation of OOS. More specifically, if it is believed that increasingly massive, powerful, expensive spacecraft represent an unsustainable path for the overall industry, and that this trend in spacecraft design will continue largely

${ }^{1}$ Corresponding Author: hastings@ @it.edu, tel:+1 6172530906 
unabated without a significant paradigm shift, will OOS play a demonstrable role in initiating and sustaining that shift? And what are the general trends in cost and design required for OOS to become a permanent fixture in space architectures, rather than a simple additional feature to existing systems? Finally, as with any new technology, there are several additional policy factors that must be considered, such as liability for damages, issues of security and transparency, the determination of operational codes of conduct, and provisions for international coordination and participation.

\subsection{Space Industry Trends}

As with almost all modern industries, spacecraft technology has progressed significantly in the past decades. One would expect that the cost and mass of the typical spacecraft would have decreased or at least remained mostly constant, even as the power and performance of each craft is improved. However, this has largely not been the case. Over the past two decades, the major trends in geosynchronous spacecraft manufacturing have been towards larger, more expensive, longer-lasting spacecraft. This is shown in Figure 1, which depicts the design lifetimes and launch masses of U.S. civil, commercial, and military spacecraft launched to geosynchronous orbit over the last two decades[1]. Similarly, it has been shown by Saleh that the cost of spacecraft also increases steadily with design life, and that cost-peroperational-day estimates exhibit diminishing returns after a design life of approximately 8 years (shown in Figure 2) [2].

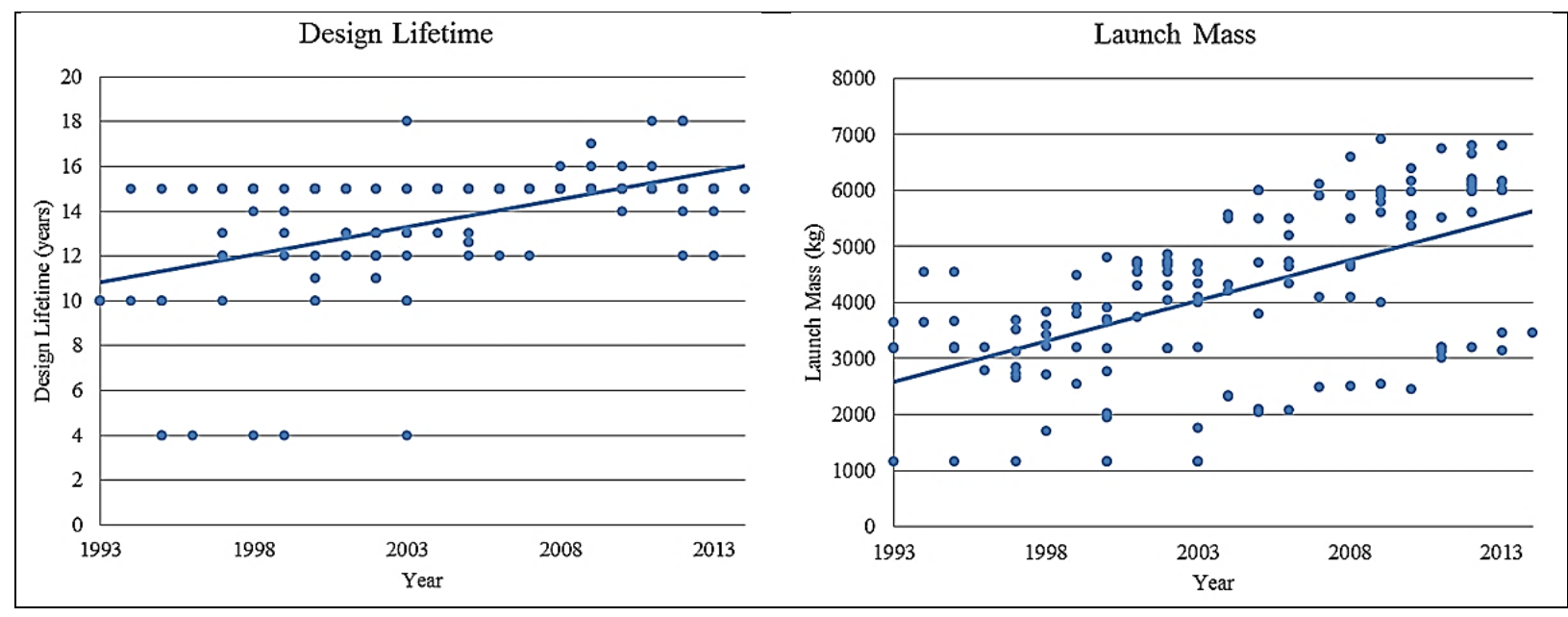

Figure 1. Design lifetimes and launch masses of active geosynchronous spacecraft over the last two decades [1]. 


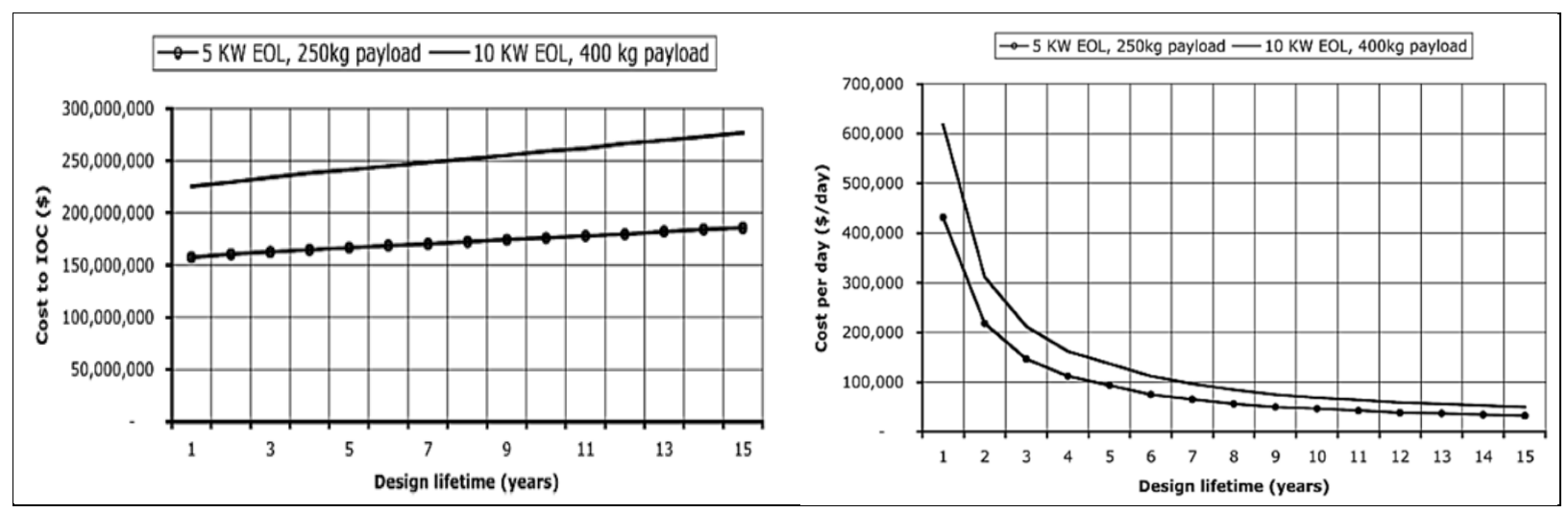

Figure 2. Cost to initial operating capability (IOC) and Cost per day (\$/day) as design lifetime is increased [2].

This is not to say that the performance of spacecraft has only held steady. In fact, the average power of spacecraft has been shown to fit an exponential growth curve as technology and customer demands have progressed over the last several decades (shown in Figure 3). Using power and mass as proxies for spacecraft performance, it is evident that spacecraft architecture designers have consistently opted for higher-performing, longer-lasting, increasingly massive space vehicles.

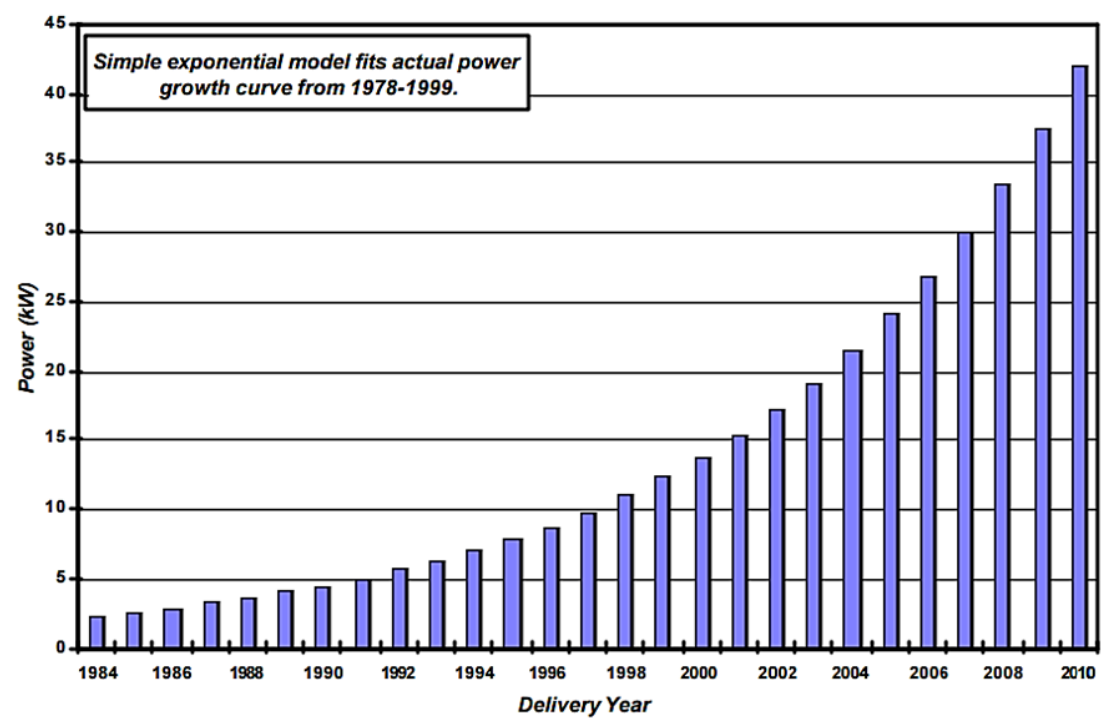

Figure 3. Increases in average power of geostationary spacecraft [3].

Current spacecraft perform exquisitely and reliably, but cracks in the overall space industry are beginning to show. Launch costs have remained stubbornly high, even as the technology behind launch vehicles has matured, and the development schedules required to build, test, and deliver new spacecraft typically extend a decade or more. Meanwhile, cost and schedule overruns on new spacecraft are the norm, and the space industry as a whole has exhibited a high degree of consolidation and monopolization as firms chase larger and lengthier contracts [4]. Proponents of on-orbit servicing often argue that spacecraft design paradigms incorporating OOS would help to alleviate some of these industry stressors, allowing for new spacecraft to halt the steady increase in mass and design life in favor of more flexible designs - ones 
which would plan for periodic refueling and/or technology upgrades [5]. Indeed, in the methodology presented below, it is assumed that an effective OOS program is one that can significantly alter long-term spacecraft design trends while also allowing for a more flexible, lower-cost industrial risk posture.

\subsection{OOS Technical Demonstrations}

To this end, several preliminary tests and studies have been conducted to examine the feasibility of onorbit servicing. One of the most prominent projects in this arena was the Defense Advanced Research Projects Agency's (DARPA) Orbital Express program, which validated the "technical feasibility of robotic, autonomous on-orbit refueling and reconfiguration of satellites" [6]. During this mission, two spacecraft were launched: a prototype serviceable satellite called NextSat and a prototype servicing satellite called ASTRO. Launched into a circular, 492-km orbit in 2007, the two spacecraft performed such operations as propellant transfer, detachment and reattachment of satellite components, and multiple rendezvous and capture scenarios[6]. The DARPA Orbital Express mission "met all of its mission success criteria" [7]; in so doing, it provided resounding evidence of the technical feasibility of on-orbit servicing, provided that the spacecraft being receiving fuel and upgraded components is compatible with the servicing spacecraft.

Another DARPA program which is currently working to validate OOS capabilities is the Phoenix program, which seeks to "develop and demonstrate technologies that make it possible to inspect and robotically service cooperative space systems in GEO and to validate new satellite assembly architectures" [8]. A key component of this program is the development of the "satlets" concept, in which a spacecraft is composed of several small individual modules that are aggregated on orbit. While some spacecraft may require specific design alterations to become capable of taking advantage of satlets and other capabilities offered, the Phoenix program is still aimed at validating an OOS concept which would not require inordinate changes to current spacecraft design paradigms.

Meanwhile, NASA has also conducted a great deal of satellite servicing tests and operations, with its Satellite Servicing Capabilities Office (SSCO) spearheading many of the efforts. Currently, one of its primary programs is the Robotic Refueling Mission (RRM), which involves testing of satellite servicing components on the exterior of the International Space Station (ISS). While the main thrust of the mission is described in the name, one thing that makes this program unique from other servicing demonstrations is that it actively seeks solutions for servicing satellites that were not originally designed for such operations [9]. Thus, RRM is working towards solutions that could potentially be applied not simply to satellites purpose-built for servicing, but to a large portion of currently operational spacecraft. In order to perform this testing, the ISS's Canadian-built robotic arm, Dextre, uses four unique tools to perform various tasks on a custom-built RRM module mounted on the ISS exterior and containing common satellite components. The RRM continues today, with new components being delivered over time and more demonstrations of on-orbit tasks scheduled, including tests involving the inspection of satellite components and experiments involving solar cells and paint materials [10].

While DARPA and NASA have proven experimentally that OOS is technically feasible, an actual operational example of the benefits of OOS is provided by the Hubble Space Telescope, which underwent five separate servicing missions to fix and improve many aspects of this well-known and long-lived spacecraft [11]. Treated less as a spacecraft and more as a floating national observatory, the Hubble Space Telescope was designed to have easily accessible and upgradeable components. It also required servicing visits by astronauts operating out of the Space Shuttle, who came ready with specially-designed tools and abundant experience gained from extensive practice runs of each servicing mission back on Earth. Thus, the Hubble does represent a special case amongst servicing missions, in which cost was largely not an obstacle and significant NASA resources were at engineers' disposal. Indeed, NASA's 2010 OOS study identified the excessive costs of Hubble servicing as one of the major contributors to the 
"myth" that servicing is necessarily a very costly, fundamentally prohibitive means of increasing space systems' performance [12].

Still, experiences with Hubble offer many lessons for future OOS operations. The fact that the entire Hubble science mission was rescued from the brink of failure by the first servicing mission speaks volumes for one of the most common rationales for developing OOS capabilities; that is, it reinforces the usefulness of OOS for fixing incredibly expensive spacecraft which otherwise could be completely lost or would experience significantly degraded performance due to some initial anomalous failure.

Furthermore, the Hubble has received significant upgrades in technology over its lifetime, and it has even incorporated scientific instruments which had yet to be conceived when the telescope was originally constructed. The Hubble's lifetime has also been significantly extended due to servicing missions, with the last mission providing it with the new batteries and other components needed to allow it to operate well into the current decade [11]. Without a doubt, the Hubble Space Telescope would be a far less capable spacecraft, as well as one which would likely have been decommissioned long ago, if it had not been designed with OOS in mind.

\subsection{Behavioral Economics Concepts}

A short discussion is needed to explain the relationship between emerging theories in behavioral economics and their likely role in the development of the space industry. One key concept at play is anchoring and adjustment [13]. This describes the way in which humans typically base their decisions on some "anchor" value, and then they adjust their estimates as new information is obtained (with adjustments typically being inadequate, due to the much larger influence of the original anchor value). Sterman showed how this heuristic causes wild oscillations in a stock maintenance game, as human players attempt to minimize a fictional firm's cost by maintaining an appropriate stock of inventory throughout small shifts in demand. Widely known as the Beer Distribution Game, players almost always become anchored to certain levels of inventory and then fail to adjust adequately as conditions change [14]. It is postulated that the same heuristic is influential in space systems designs. For instance, space systems decision-makers likely become anchored to a certain level of design lifetime, spacecraft mass, and cost per operational day, and then they adjust from those levels as conditions change. This would also provide partial explanation for why spacecraft have slowly become more massive, more powerful, and longer-lasting, as designers become anchored to the design paradigm of increasing mass, power, and design life with each generation and then fail to adjust far beyond such design preferences (even if significant cost savings and utility gains could potentially be gained through other design paradigms).

Risk also dominates the space system decision-making process. Spaceflight is an inherently risky enterprise; Wertz estimates that about $10 \%$ of launches fail to insert spacecraft into orbit, and once the craft is in orbit there is a near-infinite number of failure modes, both known and unknown that can prematurely end a space mission [4]. Space is also a field where successes largely go unrecognized, while failures often take the form of highly visible explosions or losses of critical services. And of course, a typical space mission requires hundreds of millions (if not billions) of dollars, making the stakes that much higher and giving decision-makers ample justification for avoiding any and all risks. Still, it is likely that intense risk aversion is directly linked to increasing costs and development schedules, to the point where decision-makers are overweighting the probability of mission failure and thus missing opportunities for cost and schedule reductions. This follows from one of the core tenets of Prospect Theory; i.e., that losses are felt more strongly than gains, and therefore a small chance of failure will be over-weighted by the decision-maker [15]. Furthermore, it has been shown that people are willing to spend significant amounts of money to avoid ambiguous processes (like those associated with new, unproven space systems) in favor of other processes that are known but entail a normatively equal level of risk [16]. The biases introduced by all of these phenomena can be included in a system dynamics model, by forcing extra costs and delays proportional to the perceived level of risk. These risk features also 
allow for the testing of a key rationale for the implementation of OOS: that it will reduce the level of risk aversion widely felt in the space industry and allow for decreased spacecraft costs and mass.

\section{Material and Methods}

\subsection{System Dynamics}

System dynamics modeling involves the use of interconnected loops of stocks, flows, and variables, which all together represent a larger complex system. Stocks can represent both materials and information; that is, they represent a tangible accumulation of some quantity or concept within a system. Thus, in a space industry context, stocks can represent actual satellites in orbit or under production, as well as represent the level of experience in the industry or the design choices (like mass, design life, and cost) of new generations of spacecraft. Flows, meanwhile, represent changes in the stocks, and these changes are in turn driven by the myriad variables that influence the long-term development and behavior of a system. Links are drawn between all of these stocks, flows, and variables, such that several interconnected feedback loops are formed. It is these loops which ultimately drive system behavior, as one component of the system affects another, which affects another, which then eventually comes back to inform the output of the first component. Mathematically, the stocks and flows represent a system of coupled, non-linear differential equations, which are calculated smoothly and efficiently through computer simulations. By correctly identifying the dominant links and feedbacks within a complex system, system dynamics seeks to understand overall system behavior and then inform policy decisions which can direct the system towards optimal outcomes [17]. A graphical representation of all of the concepts discussed above is provided in Figure 4 below.

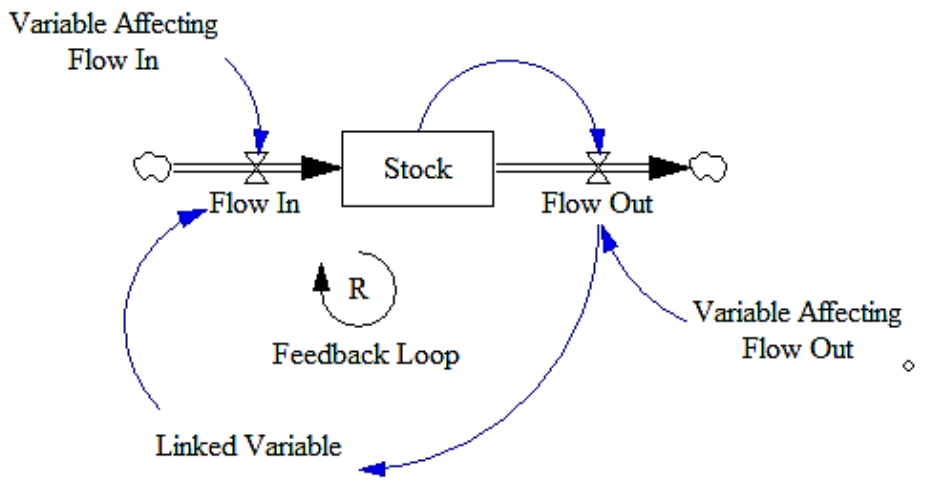

Figure 4. This simple diagram illustrates the components of a system dynamics model.

A full deductive system dynamics model, covering everything from spacecraft design to industry experience to constellation risk posture, was developed for this analysis. The construction of this model is discussed in much greater depth by Putbrese in Exploring Design and Policy Options for Orbital Infrastructure Projects [18].

This deductive system dynamics model was constructed to build and maintain a reference satellite constellation of at least 12 geosynchronous communication satellites in orbit at all times. It also continuously updates the design points for new spacecraft (launch mass, design lifetime, cost), with each new generation of spacecraft forced to improve upon the performance of the last (driven by both mass increases as well as an exogenous technology advancement curve). Meanwhile, the model tracked total industry experience, with the manufacturing of new spacecraft leading to gains in experience, while 
inactivity led to a slow degradation of experience. This experience was then directly linked to an industry learning curve, which caused reductions in spacecraft cost and production time according to the level of experience. Finally, the model determined the acceptable spacecraft risk level, using the Prospect Theory-based structure discussed further below. In summary, the model was meant to simulate the basic design points and factors affecting the manufacture and operation of a reference geosynchronous communications constellation, with an emphasis on capturing the human decision-making biases that could keep the model from returning a more "optimal," but unrealistic result.

\subsection{Incorporating Behavioral Economics Theories in System Dynamics}

Several behavioral economics concepts are a natural fit with system dynamics. For instance, the concept of anchoring and adjustment is implemented as an adjustable information stock. The anchor value is a stock that begins at a reference point; for example, in 1993 the average design lifetime of a GEO communications satellite was approximately 10 years, the average mass was approximately $2600 \mathrm{~kg}$, and the average power was about 6 kilowatts (refer to Figure 1 and Figure 3 above). As the simulation progresses, this anchor value can be adjusted, such that it changes over time according to the rules implemented by the driving variables of the structure. This reflects the way in which an anchor value can be changed slightly by one decision-maker, with the new value then acting as the anchor for the next decision-maker. For space systems, this heuristic is reflected by the fact that the performance and capabilities of current satellites will factor heavily into the expectations for future spacecraft, and thus decision-makers are more likely than not to adjust from these current anchor values when evaluating new designs.

Prospect Theory-based risk aversion is implemented using a "Success Rate" information stock, which tracks the overall rate of successful spacecraft launches and can be adjusted over time according to cost and risk preferences. The main variables governing the movement of success rate are two separate functions: a risk-based value function and a risk-to-cost function. The risk-based value function reflects the prospect theory concept of losses being felt more strongly than gains (in this case, spacecraft failures being felt more strongly than successes) [15]. This is represented by the steeply decreasing value function below the point $(0,0)$, and the more moderately increasing value function above $(0,0)$. The success rate is balanced on the other side by the risk-to-cost function, which increases the cost of each spacecraft when the success rate is adjusted above a baseline risk level (to reflect the more costly engineering required to decrease risk). This function reflects the increasing multiplier on cost when success rate is increased beyond a baseline risk of $10 \%$ (as well as the cost savings which can be accessed below this baseline risk level). These estimates for cost multipliers were determined solely to aid in the modeling of the space industry and are not considered applicable to all spacecraft types. The success rate reaches equilibrium when the value function and risk-to-cost function reach an equivalent level of adjustment, reflecting the assumption that decision-makers will decrease risk only to the extent allowed by cost considerations (i.e. when prospect theory utility is maximized). Finally, the impact of the value function is tied to the amount of time required to produce replacement spacecraft, reflecting the fact that decision-makers will be far more risk averse when they know that any failure will require years to replace. 


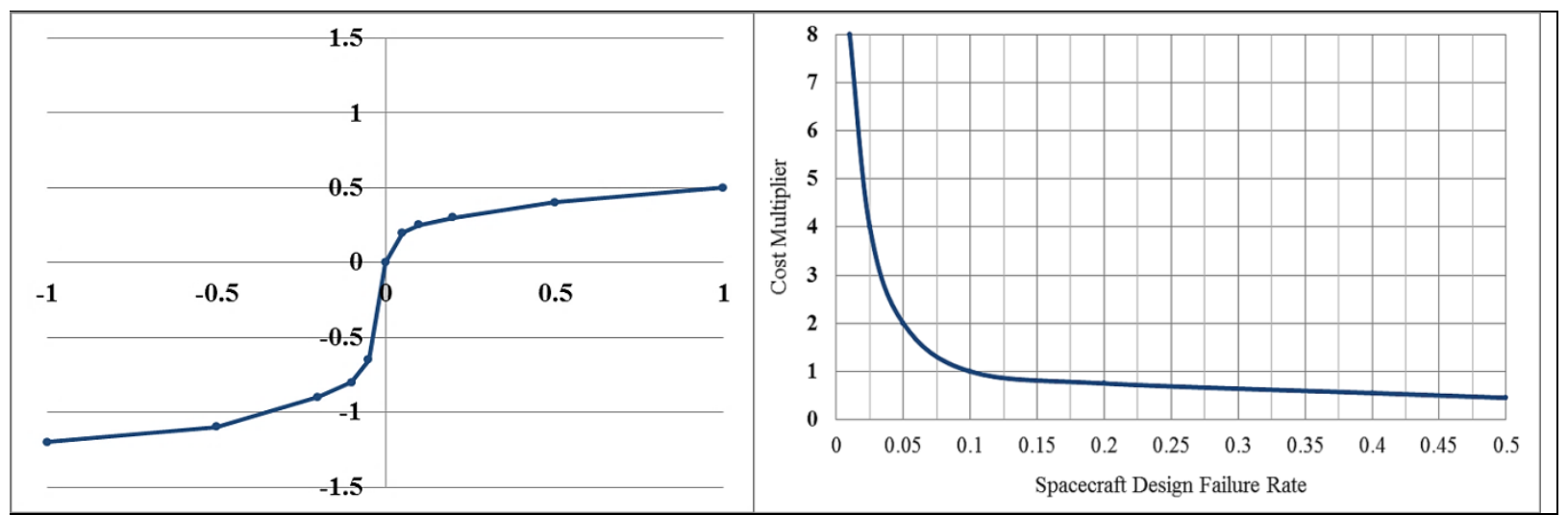

Figure 5. The graph on the left represents the Value Function, and the graph on the right represents the Cost Multiplier applied to lower risk levels.

\subsection{Using System Dynamics To Assess On-Orbit Servicing Effects}

To test the wider effects of the introduction of servicing spacecraft to overall space architectures, the system dynamics model was tuned to simulate a pre-servicing/post-servicing industry evaluation. This was achieved primarily by running the model until the simulated space industry resembled present day paradigms, as shown by the space vehicle (SV) mass and design life closely resembling the real-world progression of geosynchronous communications spacecraft. This also served as a means of validating the model's performance, and the results of this test are shown in the figures below. As is shown, the model returned SV mass and design life values very closely resembling that of real world satellites, lending a degree of confidence to the model's ability to predict overall space industry and operational effects into the future.

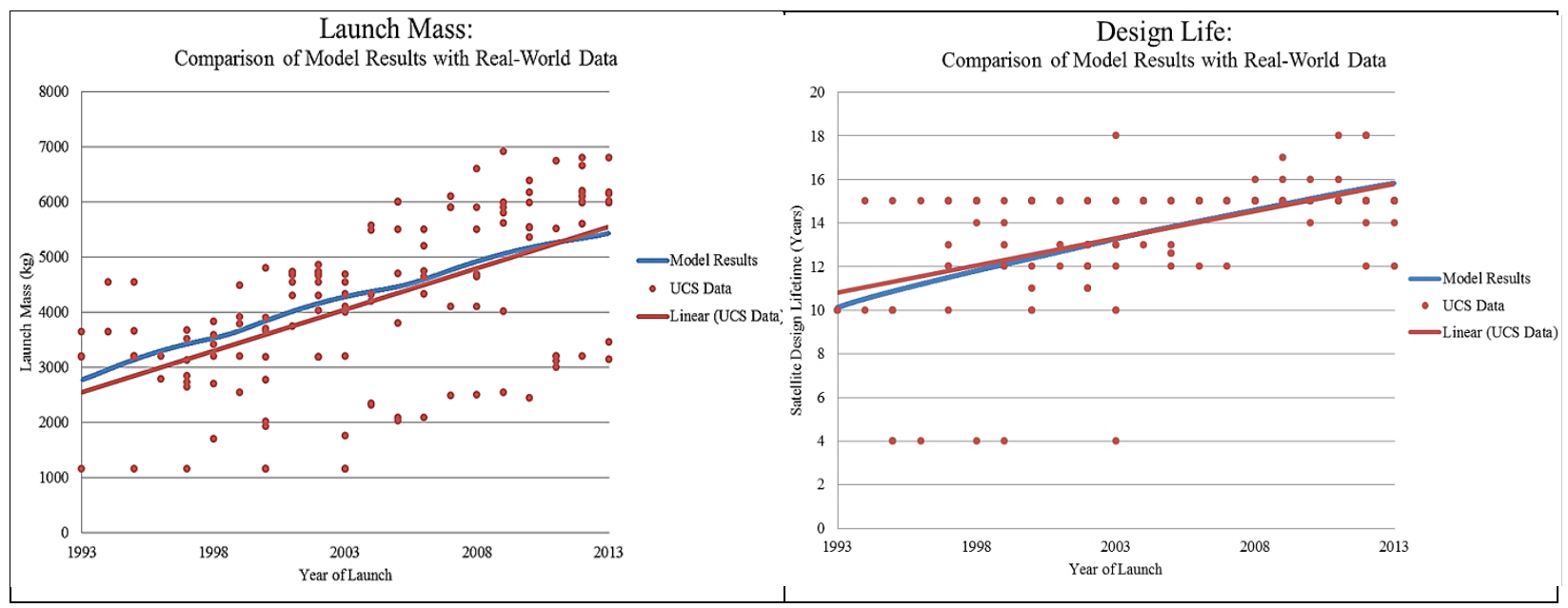

Figure 6. The above graphs for satellite launch mass and design life show that the model returns accurate results for the progression of these key design variables. The effects of OOS implementation were modeled directly for an additional 30 years after these results (i.e., from present conditions until approximately the year 2040). 
Then, with model parameters resembling those of present day satellite architectures, a significant reduction in the architecture's risk aversion level can be examined, as spacecraft operators begin to rely upon the repair and repositioning capabilities of the servicing infrastructure. This shift in risk posturing is forced through manipulation of the prospect theory-based value function, such that designers become willing to accept more risk (i.e., feel less negative emotion from failures) in the interest of cost savings and greater use of the servicing infrastructure. The shift in the risk function is applied in tandem with a series of new pricing structures: free, partial, or full price of a replacement satellite, applied over all spacecraft produced. All of this is meant to alter the overall "attitude" of the model from one of high risk aversion to one which is more moderate risk aversion, given a relatively "cheap" reduction in risk from reliance on the servicing infrastructure and the cost savings associated with building less reliable spacecraft.

It must be noted that only SV launch mass and design life were tuned to resemble present day values. Another key variable, that of Total Program Cost, is meant to track the progression of the sum of all the major costs for the launching of the reference constellation, including manufacturing, launch costs, any added servicing fees, or for the replacement of failed spacecraft (with spacecraft cost estimates being derived from cost models provided in Space Mission Engineering: The New SMAD [4]). However, the actual monetary values generated for this variable are not meant to be reflective of real-world figures. Instead, what this model can show are the general, overall trends in costs which can be expected to result from OOS implementation.

Furthermore, the "SV Construction Learning Curve" stock and flow chain is more complex than the other variables. It begins at an initial level of 1 (representing full price, full production time), and is then decreased by experience gains (down to a minimum level arbitrarily set as .2 or $20 \%$, such that it could never be so low that space programs would effectively be modeled as free). Gains in industry experience, immediately caused by the launching of new spacecraft, drive reductions in the Construction Learning Curve, which then acts as a multiplier to individual SV Cost and Production Time (such a lower value for the learning curve translates to lower costs and faster production). However, industry experience is also slowly eroded over time due to entropy, or the slow degradation of human capital and the effects of technological obsolesce. The model is tuned to expect two generations of technology advancement per decade; specifically, after 10 years, knowledge is only worth $25 \%$ of its original value with respect to current activities. The model is also tuned such that the marginal gain in knowledge over time decreases with each individual unit (e.g. the cost and production time decrease between the second and third SV constellation build is substantially more than between the $52^{\text {nd }}$ and the $53^{\text {rd }}$.) In summary, the structure tracks the number of satellites which are produced within a time period, and the efficiency at which this knowledge is captured by the industry, in order to determine the impact of this activity via gains in the industry learning curve and subsequent decreases in satellite cost and production time. For a deeper discussion of the system dynamics model construction, refer to Putbrese's 2015 thesis [18].

To assess the long-term effects of OOS incorporation into the reference customer space architecture, three main scenarios were devised and simulated. The first is a future scenario where OOS has no effect on overall spacecraft design trends; i.e., satellite mass continues to increase with each generation as architecture designers attempt to pack more and more performance-increasing components (more transceivers, larger fuel tanks, larger solar panels, etc.) on each new satellite. In this scenario, OOS is still modeled as easing the designed-for success rate of new satellites, as there is much less of a drive towards risk aversion from the occurrence of spacecraft failures, due to the ability to easily rescue or repair onorbit failures. However, this rescue and repair of failed spacecraft is considered to be the only function that OOS serves in this scenario, as the continued increase in satellite mass indicates that spacecraft operators are not conducting such OOS operations as periodic refueling or technology upgrades; i.e., OOS is used only sparingly, largely for emergency operations, and is not heavily incorporated into customer architectures and thinking. 
The second scenario is one in which satellite mass is held constant after OOS is implemented. This may be the most realistic scenario, as spacecraft designers are likely to determine that any mass savings they achieve through the use of OOS would simply allow for the inclusion of more components. This scenario is supported by the case of all-electric spacecraft, which were originally pitched as a means of freeing up significant space for extra communications capability due to their lack of chemical propellant. In addition, while each all-electric spacecraft does weigh less than a chemically-propelled counterpart, the mass savings are somewhat negated by the time of launch, as the spacecraft operator chooses to simply launch more of them to increase overall constellation performance [19]. Thus, in the mass-constant scenario, it is assumed that in addition to the rescue and repair of spacecraft failures, OOS is also used for limited refueling and upgrading operations (in other words, in a more operational and dependent manner than in the first scenario). Holding mass constant in this scenario was forced exogenously, in order to assess the resulting trends in other industry variables (such as SV design life, total costs, and industry experience) within a narrower future scenario.

In the third and last scenario, the implementation of OOS leads to steady mass decreases in satellite mass, as spacecraft operators rely more and more on the refueling and upgrade capabilities offered through OOS. In this scenario, not only would the spacecraft be carrying less fuel, but it is also assumed that several of the on-board components would be lighter and less enduring, as the satellite receives continuous, regular technology upgrades and improvements over its design life. Additionally, the spacecraft operators become even better over time at capitalizing on the capabilities of OOS, perhaps through such techniques as the modularized "satlets" concept currently being explored by DARPA, where a common satellite bus would be designed for periodic upgrades and the addition of new, standardized performance modules [20]. This is considered to be the best - though perhaps not the most realisticscenario for OOS implementation, as the reference space architecture is considered to be making the greatest use of OOS and incorporating its capabilities heavily in long term operations. Indeed, in a massreduction scenario such as this, it is assumed that the constellation is critically reliant on OOS as a means of maintaining overall performance throughout satellite design lives. Like the previous scenario, these mass reductions were forced exogenously in order to assess long-term trends in other industry and architecture variables.

Finally, in order to model the potential costs of OOS, four basic cost structures were applied in each scenario based on current cost models for space and terrestrial infrastructures. The "No Cost" case represents an infrastructure where use of servicing does not cost anything to the customer. This case is analogous to the GPS constellation, which provides all users with continuous navigation and timing services at zero cost (provided the customer already has the necessary technology to make use of the GPS signal). The "Low Cost" case represents a servicing fee of $10 \%$ of the normal cost of a spacecraft. In other words, the extra cost associated with using the infrastructure is priced at $10 \%$ of what it would cost to simply replace a spacecraft, applied across all spacecraft (as it is assumed that all will be designed to utilize the OOS infrastructure during their design lifetimes). The Low Cost case is similar in concept to toll roads, in that a small fee is incurred to use the infrastructure but it is low enough to be marginal compared to total costs. The "Moderate Cost" case represents a servicing fee of 50\% of spacecraft cost, and it is meant to simulate a more expensive infrastructure funding structure, similar in nature to airport landing, refueling, and parking fees. Finally, the "High Cost" case represents a servicing fee of $100 \%$ of the cost of a spacecraft, which is meant to represent the extreme end of feasible servicing costs; any higher and it would no longer make economic sense to service a spacecraft rather than simply replace it. In all cost structures this fee is applied across all new spacecraft, as it is assumed that all satellites will be designed with the expectation of at least some servicing within their design lives. It should also be noted that, with the servicing fee being applied to the spacecraft in the system dynamics model, spacecraft failures can then be diverted directly back into the production pipeline; i.e., the cost of rescuing a failed spacecraft is covered in the architecture design loop, and the model assumes that all failures will be automatically rescued, such that the constellation continues to maintain its minimum capability 
requirements. Of course, in reality OOS may not be so assured and also may only be paid for as it is required, but this analysis assumes that servicing is completely integrated into the reference space architecture, and thus a fee across all spacecraft is reasonable.

In all three scenarios, the following key variables are shown over a future thirty-year life span: SV Launch Mass, SV Design Life, Total Program Cost, and SV Construction Learning Curve. In all cases, the model is first run until SV launch mass and design life match present-day averages, and then OOS implementation effects are modeled into the next 30 years. This was done to ensure that the model is "primed" at the start to match current paradigms, and then must adjust from these present-day anchors as OOS effects take hold [13]. Then, from this point on the model evaluates the progression of large-scale industry and architecture trends, with all cases being compared to a case in which no OOS effects are enacted. What is of most value from the model results are not the specific figures themselves, but rather the overall trends caused by OOS. This is due to the original research question, which asked for an evaluation of the necessary large-scale conditions in space architectures and the industry in general to ensure long-term viability of an OOS infrastructure.

\section{Results}

The key results of the first scenario, in which the implementation of OOS has no effect on overall spacecraft mass trends, are shown below in

Figure 7.

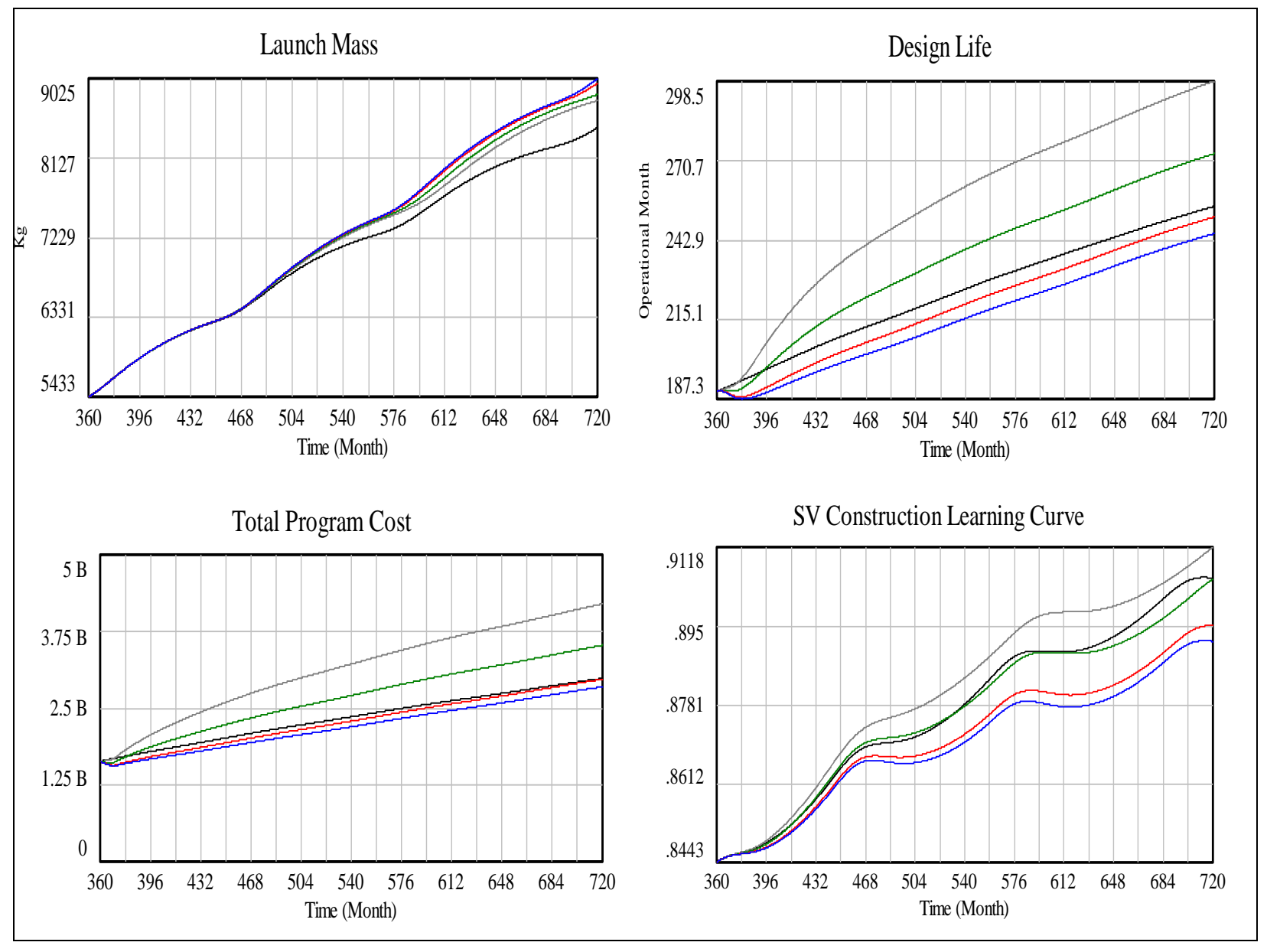


Figure 7. Results of the increasing spacecraft mass scenario. The blue line represents a No Cost OOS infrastructure, the red line represents a Low Cost infrastructure, the green line represents Moderate Cost, and the gray line represents High Cost. The black line displays the results of the Steady State results of the model without OOS implementation.

In the launch mass and design life parts of Figure 7, we see that the trend of increasing launch mass and increasing design life continues unabated with a small effect of the OOS in the later years. Of course, in the high cost case the total program cost is the highest. For all cost cases, though, the SV Learning curve continues to increase over time, which means that industry experience continues to erode as spacecraft live longer on orbit.

Next, the results from the constant-mass scenario, in which spacecraft mass holds steady at its modeled value when OOS is implemented, are shown in

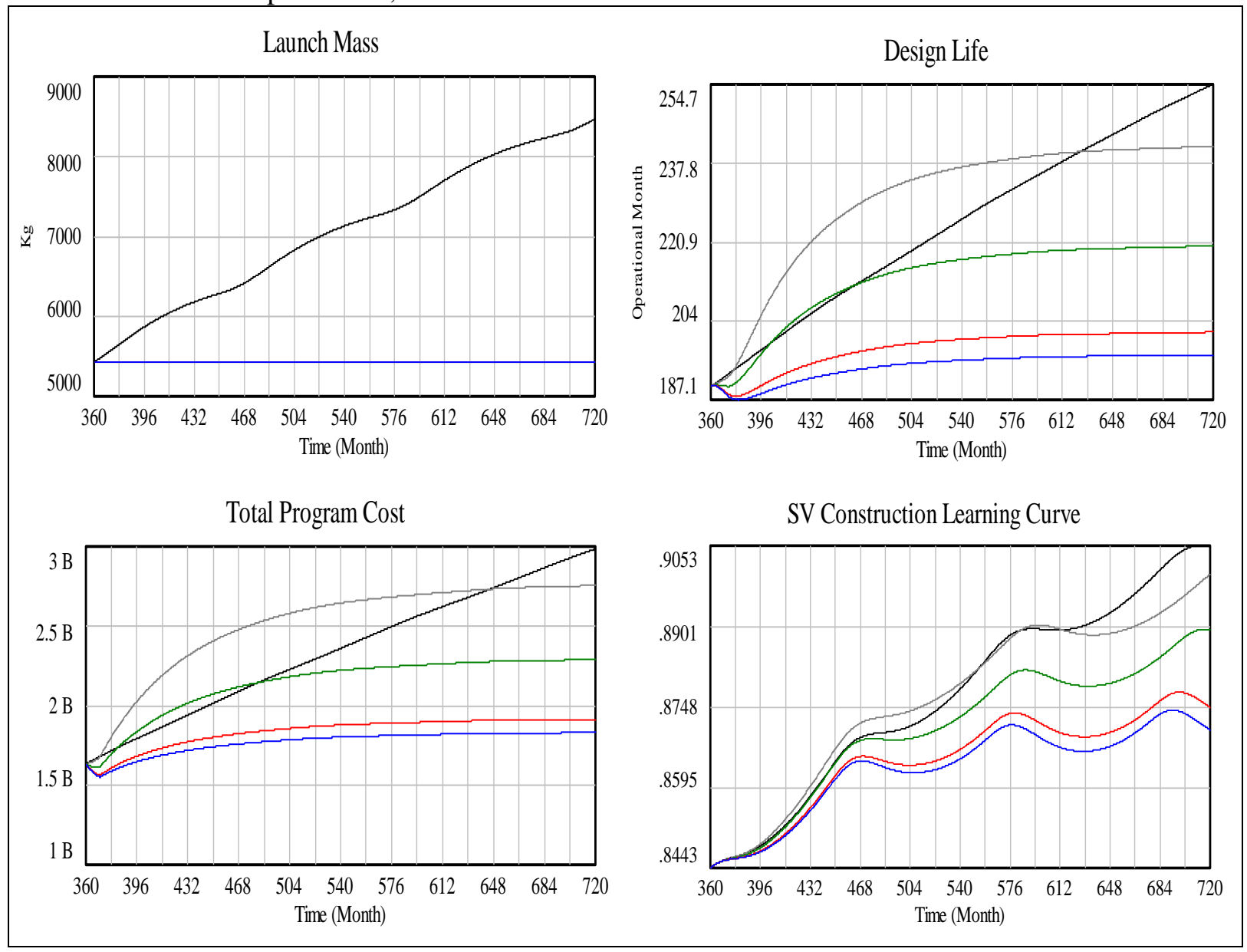

Figure 8 below: 


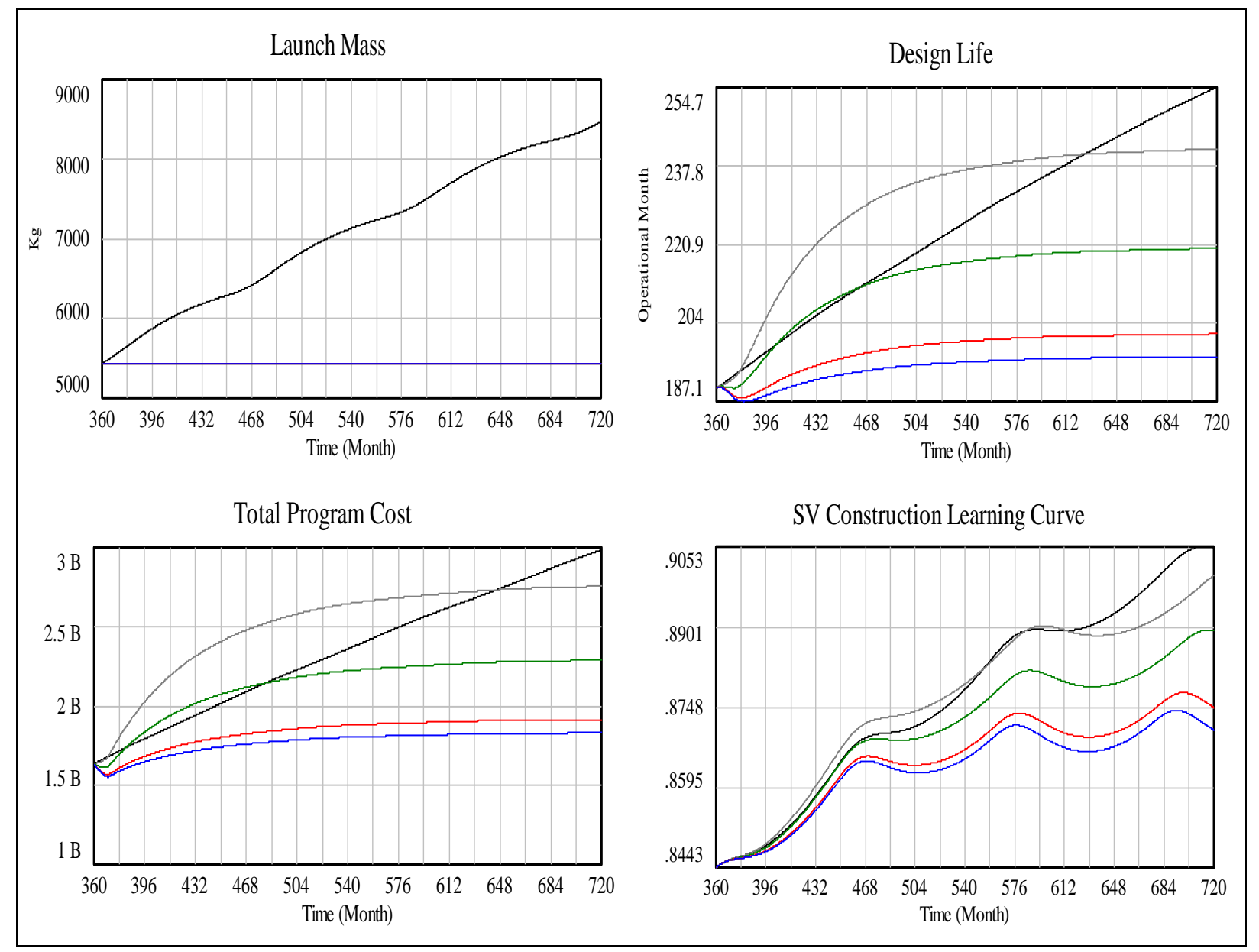

Figure 8. Results of the constant spacecraft mass scenario. The blue line represents a No Cost OOS infrastructure, the red line represents a Low Cost infrastructure, the green line represents Moderate Cost, and the gray line represents High Cost. The black line displays the results of the Steady State results of the model without OOS implementation.

In this case, the launch mass is held constant and the design life in all OOS cost cases abates at some level after approximately two decades. This then leads to lower final levels for program costs and learning curves, although in all cases it is unlikely that trends would actually reverse direction.

In the third scenario, steady reductions in spacecraft mass result from OOS implementation, as servicing capabilities are incorporated more and more into the reference architecture. The results from this scenario are shown in 


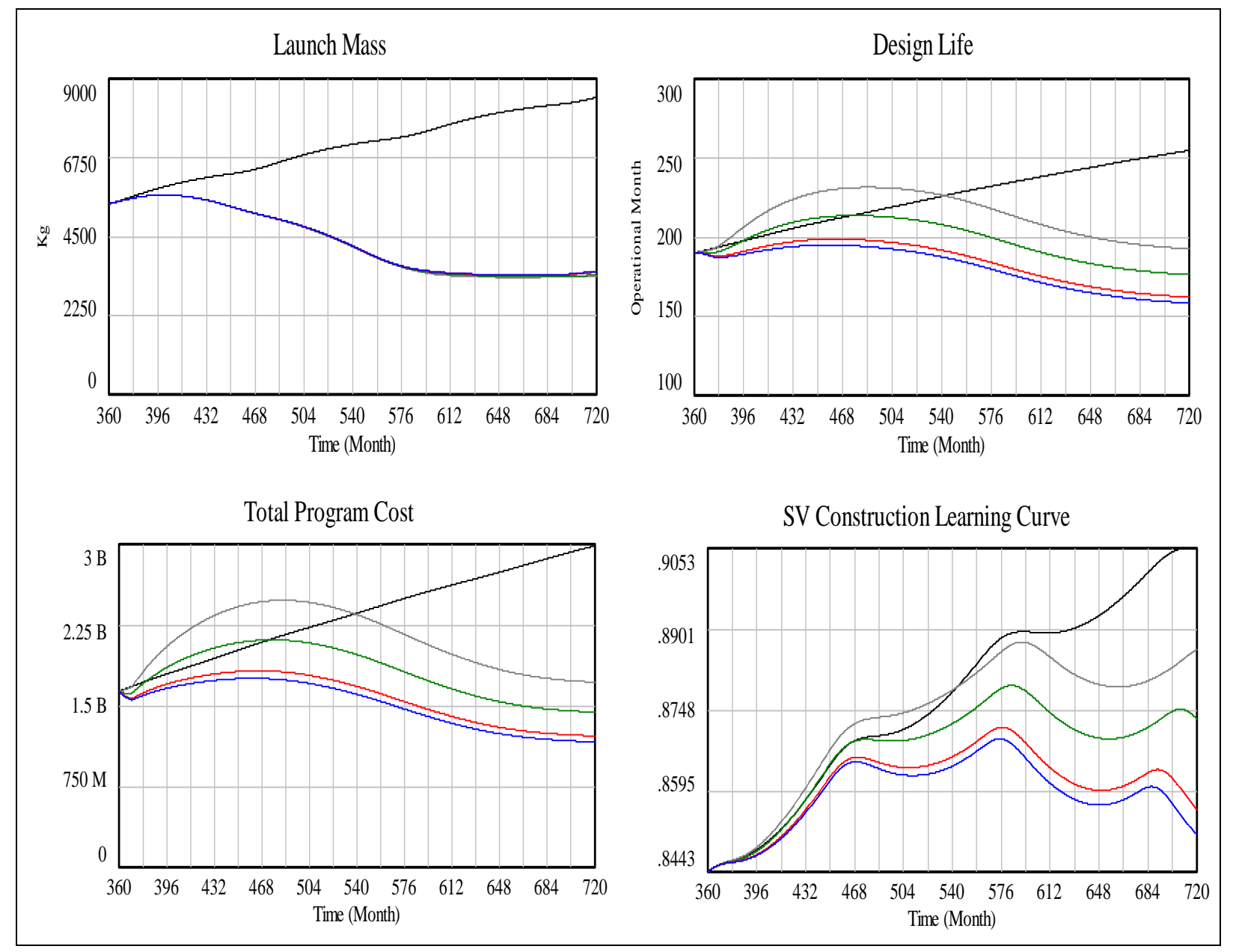

Figure 9 below.

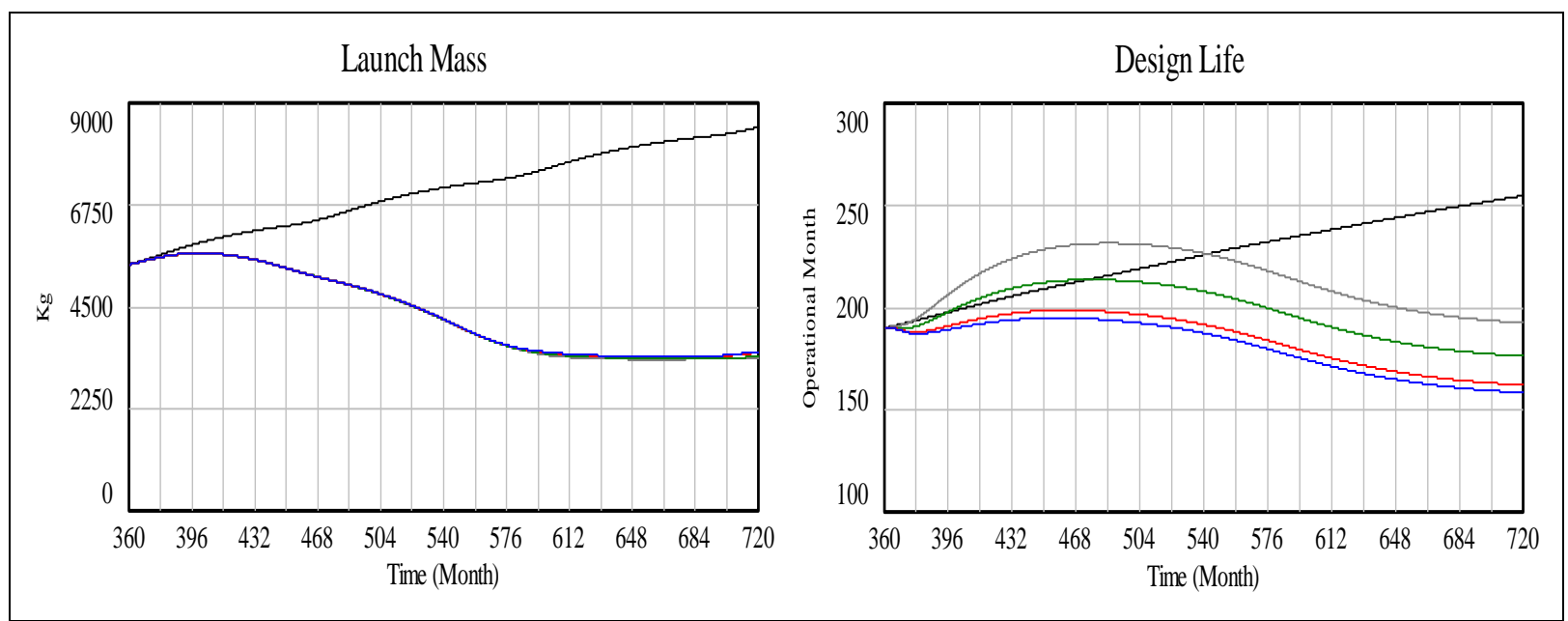




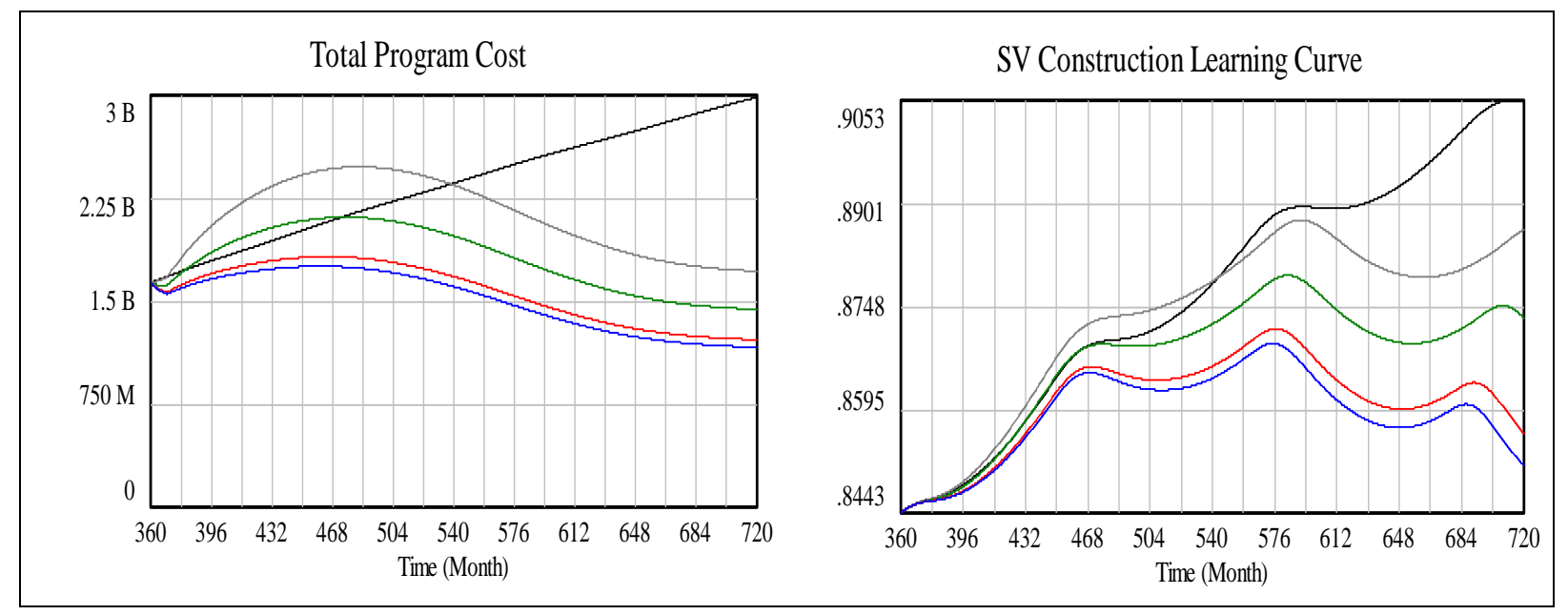

Figure 9. Results of the decreasing spacecraft mass scenario. The blue line represents a No Cost OOS infrastructure, the red line represents a Low Cost infrastructure, the green line represents Moderate Cost, and the gray line represents High Cost. The black line displays the results of the Steady State results of the model without OOS implementation.

In the mass decreasing case, which envisions full incorporation of OOS capabilities, design life now decreases, which leads to substantially reversed trends in total program costs and industry learning, especially in the Low and No Cost cases.

\section{Discussion}

\subsection{Analysis of Results}

The three scenarios analyzed above reveal a few consistent trends with regards to OOS implementation into customer architectures. First, the most intuitive conclusion holds firm: that for the greatest chance of sustainability, OOS will need to be offered at the lowest cost possible. While this is an obvious conclusion, this is not to say that OOS would not be found valuable at higher prices, or that this analysis captures all of the value provided to customer architectures through servicing. Long et. al., for example, demonstrate one way that OOS provides value in the form of increased system flexibility and other system "ilities" (for example, expandability) [5]. Indeed, even in the first scenario, where satellite mass, design life, and total program costs continue to increase over time, the No Cost and Low Cost cases were at least relatively similar in cost to the base case (if a bit cheaper), and this does not reflect the significant value to be found in cases where OOS would be rescuing failed spacecraft or allowing useful spacecraft to be refueled and extended in operational lifespan. Thus, a low cost OOS infrastructure, even one which is used sparingly and only in relative emergencies (as the first scenario envisions), still provides benefits to the overall industry; it just may not be addressing more of the core issues facing the industry.

This leads to the second conclusion, which is that, in order to spur wider benefits throughout the space industry, the capabilities of OOS will likely need to be incorporated substantially into customer architectures. In other words, for OOS to be successful in the long term and drive the industry towards more sustainable paradigms, it will need to be embraced in such a way as to cause fundamental shifts in design and constellation risk management. For example, to extract maximum value from the OOS infrastructure, spacecraft would likely have to be launched without a full lifetime's worth of on-board fuel, or with components that are expected to be replaced periodically with upgraded technologies (as for 
example, happened with the Hubble). This represents a significant departure from current practices of launching spacecraft without any plans for future changes or maintenance (beyond software updates), and spacecraft operators may be reluctant to make such drastic changes to their architectures. However, this analysis does support the conclusion that, should space architectures make the necessary alterations to fully incorporate OOS, they can expect to see long term value and lasting benefits to the overall industry. It is also reassuring to note that NASA's On-Orbit Satellite Servicing Study similarly concluded that, as customers rely more and more on OOS capabilities, then they should expect to see cost reductions at the mission, program, and agency levels [12].

Furthermore, the progression of the three scenarios shows that as OOS is incorporated more and more into customer architectures, then they are able to tolerate slightly higher prices than if OOS is only used sparingly. Of course, in all three scenarios, the High Cost cases were viewed as unlikely to drive long term sustainability; indeed, if OOS is very expensive, then spacecraft operators are likely to simply replace failed or under-performing satellites rather than pay to have them serviced. However, in the second and third scenarios, it was shown that customer architectures are likely to be willing to pay at least at the Moderate Cost level, compared to the cost of the base case which does not bring with it any of the other benefits associated with OOS. Recalling that the Moderate Cost cases applied a servicing fee of $50 \%$ of the base satellite cost, this does represent a relatively high cost tolerance in exchange for the capabilities offered through OOS. Once again, this does assume that servicing is both assured and in the critical path for the customers, and this will almost certainly require effective policies in place to govern and support an OOS infrastructure (which is discussed in-depth in the following sections).

Along these lines, the results suggest that the determination of specific "break-points" beyond which OOS is no longer feasible will vary significantly with the level of OOS implementation envisioned. While greater incorporation of OOS capabilities is linked to an overall higher cost threshold, this analysis does not attempt to capture the value of OOS to more specific customers or space architecture types; e.g., a large constellation of standardized, long-lived communications spacecraft at geosynchronous orbit may be able to extract more value from OOS than a smaller, simpler, short-lived imagery spacecraft in low-Earth orbit. Thus it will largely be up to individual customers to determine their own willingness to pay for servicing; indeed, Saleh has already done much to quantify the value of OOS to customers, when considering the increase in options and system flexibility that servicing capabilities bring to space architectures [21]. However, if OOS delivers on its promises of significantly reducing spacecraft risk of failure, fuel depletion, and/or technological obsolescence, then this analysis does support the conclusion that customers would be willing to pay a significant amount as they incorporate OOS capabilities more and more into their architectures.

The small difference in benefits between the No Cost and Low Cost cases further supports the assertion that servicing fees are likely not as influential on long-term sustainability as is the level of overall incorporation of OOS capabilities. If the No Cost case was shown to be significantly more powerful in driving down program costs and satellite design lifetimes, then it could be said that customers are highly sensitive to any additional costs and would be unwilling to utilize an infrastructure that comes with any extra fees. However, from these results, it is suggested that customers would only become averse to OOS if it priced quite high (approaching the cost of launching entirely new spacecraft). This is especially true in the cases where the customers are actively making design changes to better incorporate OOS capabilities. Thus, while these results show that a low cost infrastructure is inherently more beneficial than a high cost one, this does not mean that OOS has to be developed and operated under regimes that offer it completely freely; rather, small fees can be applied without fear of significantly hampering longterm sustainability.

Perhaps the largest drawback of this analysis, and the greatest hindrance to its generalizability to other cases, is the fact that it is heavily focused on large, relatively standardized commercial communications 
satellites typically found at geosynchronous orbit. While these types of satellites are common and represent a large portion of all commercial space operations, they are far from being the only kinds of spacecraft to which an OOS infrastructure would render services. At the same time, however, the conclusions generated from these results are considered to be widely applicable to other infrastructure projects and space systems. For instance, to incentivize participation in any infrastructure, it is almost always necessary to offer its services at relatively low cost. Furthermore, simply lessening the level of risk aversion in the space industry, whether through OOS or another means, is beneficial but also unlikely to significantly alter dominant design trends.

Finally, as with most any model, the results become less and less reliable the farther one projects into the future. This model seeks to temper this somewhat by focusing on "big picture" variables, such as average satellite design life and launch mass and the accumulation of industry experience, and the results generated are meant to inform large-scale policy regimes, rather than more specific system design points. However, it is impossible to know for sure if a model applicable to today's space industry will remain relevant to the space industry 30 years from now. Indeed, any number of innovative technologies or shifts in stakeholder values could result in a much different commercial space environment than the one which was simulated by this model. However, within the context of analyzing the effects of general shifts in spacecraft costs and risks which may result from an infrastructure project like OOS, these results do provide a few meaningful conclusions which policymakers must consider in the development and launch of such a system.

\subsection{O0S Infrastructure Policy Recommendations}

It is expected that a fleet of OOS spacecraft, involved in regular servicing operations and interacting with a variety of customer spacecraft, would require significant policy development to be economically viable and deal with the substantial legal and liability issues involved in interacting physically with customer satellites.

As shown in the system dynamics modeling results, for the greatest chance of long term viability and success, an OOS infrastructure should be offered at the lowest cost possible (that is, at the lowest cost to the customer). However, the costs to fully develop, launch, and maintain an effective OOS infrastructure are expected to be quite high. For example, NASA's On-Orbit Satellite Servicing Study concluded that a relatively simple mission, involving the autonomous capture and repositioning of 10 customer satellites in geosynchronous orbit, is estimated to cost approximately $\$ 910$ million (which includes all development, manufacturing, and programmatic costs) [12]. Meanwhile, a refueling mission capable of providing services for up to 25 customer spacecraft is estimated to cost over $\$ 1$ billion, while an even more complex assembly mission is likely to cost over $\$ 3$ billion [12]. Thus, for such an infrastructure to be developed and funded adequately - and then to have low enough prices to incentivize customers to actually use itsignificant upfront investment by public agencies would likely be required; investments which may or may not actually be fully recouped by the investor, at least in strictly monetary terms. The need for significant upfront investment is especially cogent when considering the fact that there are as yet no readily available customers in line to pay for servicing operations, making for what many describe as a "chicken-and-egg" problem of needing both an established infrastructure as well as customers ready to use the infrastructure [22].

Luckily, agencies like DARPA and NASA have already performed much of the initial testing and experimentation required to demonstrate the technical feasibility OOS. Following from this, there are a variety of policy regimes that could now take OOS from the experimental stage to a more operational footing. Perhaps the most straightforward policy would be to develop OOS as a civil project, launched and operated by an agency such as NASA. In this scenario, the infrastructure would be developed 
primarily for use by the agency, to conduct servicing operations of its own assets, with the option to have other entities pay for use of the infrastructure (similar to how NASA's Near Earth Network is currently operated) [23]. There are several advantages to such a regime; for example, maintaining the infrastructure as a civil project can help to ensure adequate funding (especially once the infrastructure is established), as well as allow for lower costs than if the infrastructure had to generate a profit. Potential customers would also be likely to have more confidence in the performance and safety of an infrastructure provided by such an agency as NASA, due to its expertise and relative transparency. However, the main drawback to this regime is that it may be quite difficult to secure the substantial funding required to turn NASA's servicing experiments into an established infrastructure. One could also raise the argument that a purely public infrastructure may be lacking in the efficiency and cost-effectiveness that could result from a more private venture.

A similar policy regime to fund and develop an OOS infrastructure would be to initially launch the system as a defense security architecture, with its primary mission being the servicing of national security assets. This would be operated and maintained similar to the GPS constellation, with the U.S.

Department of Defense retaining ownership of the infrastructure but offering its services to others as its scheduling allows. Advantages to this policy are much the same as those of the civil project described above, in that the infrastructure could draw on the substantial defense budget to pay for its development and allow for lower usage fees, as well as benefit from the decades of experience in military space operations. The main drawback to this policy, however, is that the infrastructure would inevitably be viewed as a military project; indeed, the potential for OOS capabilities to be weaponized is already viewed as a major concern [24]. Thus, customers may be more averse to participation (especially international entities), and objections could be raised on the basis of the Outer Space Treaty's strict prohibitions against weaponization. The likelihood of such a scenario is already being evidenced by other nations' development of their own satellite navigation constellations, even though the official U.S. policies governing GPS promote transparency and international interoperability [25]. In summary, while operating an OOS infrastructure as a national defense asset offers many advantages in funding, oversight, and expertise, it is also likely to have more substantial political and legal impediments.

Another policy framework for OOS implementation is that of the public-private partnership. Under this regime, a public agency such as NASA or DARPA plays a large role in the management and overall guidance of the infrastructure, while allowing private firms to propose, develop, and operate their own systems. This framework also allows for large public investment in the initial development of the infrastructure, in addition to assuring private firms that civil or defense architectures will be available as initial customers. The Space Act Agreements currently governing NASA's procurement of commercial crew and cargo operations from private firms are a current example this sort of public-private partnership, which seeks to capitalize on the efficiencies and innovations of private industry while also drawing on the experience and oversight of public space agencies [26]. The largest drawback of such an arrangement is that private space systems may be viewed as slightly more risky and less proven than public systems, but this perception of risk can be largely mitigated through transparency and the demonstration of operational performance before more public funding is awarded. Public agencies must also be willing to relinquish a significant amount of operational control of the infrastructure, and there must also be clear regulations for ownership and liability governing all servicing activities.

The public-private partnership framework appears to offer the greatest likelihood of success and realworld implementation, and this is evidenced by the fact that DARPA has recently promulgated a Request for Information (RFI) along these very lines. Specifically, the RFI states that "DARPA is particularly interested in establishing a public-private partnership that would make cooperative robotic servicing available to both military and commercial GEO satellite owners on a fee-for-service basis. The partnership would help develop near-term technical capabilities and significantly contribute toward the creation of a sustainable, commercially owned-and-operated space robotics enterprise" [27]. From this 
statement, it is clear that an OOS infrastructure is intended to not only undergo initial development as a public-private partnership, but it should also one day be spun off as a completely commercial venture (albeit with civil and defense entities as primary customers, as many commercial space architectures currently operate).

Finally, the results from the analysis in this paper do support the conclusion that a public-private partnership can ensure long-term sustainability of not only an OOS infrastructure as long as customers have the necessary degree of confidence to begin making the required design changes to their architectures to begin to more heavily incorporate OOS capabilities.

Regardless of the policies governing the funding and development of an OOS infrastructure, there are in addition several political and legal considerations that would accompany such a project especially if there were international cooperation. Of course, the foundations for international cooperation and coordination in space have already been in place for nearly half a century, as codified in the 1967 United Nations Outer Space Treaty. This document laid out in broad terms the vision for space as a highly international arena, in which no nation can claim sovereignty and all parties are compelled to act in such a way as to promote peaceful, transparent relations and space activities. The UN Outer Space Treaty also injects a degree of liability and ownership into the governance of space, by stating that states retain control over all objects they launch, or procure the launch of, into outer space, for as long as they remain in outer space, and that states can be held internationally liable for any damage caused by their space objects [28]. Thus, it is expected that any new policy regime for the conduct of OOS operations would expand upon and further clarify the provisions already agreed upon in the Outer Space Treaty.

In an extensive overview of the issues facing OOS implementation, Belcher et. al. identified six key policy areas which could impede future servicing projects: Responsibility and Ownership, Insurance, On-Orbit Operational Regulation, Assurance, Spectrum and Slotting, and Imaging [24]. These issues are important for future work and will need to extensively developed in the context of international law in order for OOS implementation to be viable.

\section{Conclusions}

The primary conclusion from the system dynamics analysis of the implementation of an OOS infrastructure is that, for the greatest chance of long-term sustainability and benefits from the infrastructure, customers must be willing to make significant architectural design changes to fully incorporate OOS capabilities. It was shown that a shift in risk attitude alone from the use of OOS was not enough to reverse the upwards trends in satellite mass and design life in a system dynamics model simulating a theoretical constellation of geosynchronous communications satellites. Indeed, in such a case where designers continue to increase spacecraft mass generation after generation, as they have for the last several decades, then the added costs of OOS may actually exacerbate industry trends and further degrade industry knowledge.

More or less freezing spacecraft mass at current levels as OOS is implemented was shown to largely halt potentially unsustainable industry trends, but in order to actually reverse trends and return spacecraft design points to more sustainable levels, spacecraft designers must be willing to make significant alterations to architectures. Such changes would likely include much smaller fuel tanks or less robust components, as constellations begin to rely on the OOS infrastructure for periodic refueling and technology upgrades.

It was also shown that OOS should be offered at as low cost as possible to the customer, as this allowed for the greatest benefits in design trends and overall sustainability. However, OOS does not necessarily 
have to be completely cost-less to the customer; indeed, as the customers incorporate more and more OOS capabilities into their architectures, the results suggest that they are then able to tolerate moderately higher servicing fees. Furthermore, the cost of the infrastructure was shown to be a much less significant factor in the success of OOS than the overall level of incorporation of servicing capabilities into spacecraft design and operations. This result should be reassuring to proponents of an OOS infrastructure, as the tolerance for servicing fees means that there are several viable options available to policymakers for the ultimate development of the project.

Along these lines, several options for the funding and development of OOS were outlined and discussed. There is a much greater likelihood that OOS will be implemented through a public-private partnership, where public agencies provide much of the funding and oversight, while a private firm conducts the manufacturing and operation of the infrastructure. Indeed, the groundwork for such an arrangement is already in place; NASA and DARPA have already conducted much of the initial development and testing of OOS technologies, and DARPA has recently issued a request for information from private contractors interested in the many aspects of an OOS project [27].

Finally, several policy and legal issues surrounding an OOS infrastructure were introduced, with the six main areas of concern being responsibility and ownership, spacecraft insurance, on-orbit operational regulation, assurance of non-weaponization, spectrum and slotting, and imaging regulations [24]. For the overall framework for OOS policy, it is asserted that regulation of the infrastructure may borrow from the UN Outer Space Treaty.

Future work in the assessment of OOS concepts could focus on more specific price points for successful implementation. For instance, Saleh et. al. have examined the value OOS provides to space architectures in the form of flexibility, demonstrating that it is likely that the ultimate rationale for an OOS infrastructure will not lie in a strictly cost-to-performance basis [21].

For an OOS infrastructure, much of the vision for implementation has already been realized. NASA and DARPA have successfully conducted the initial technical demonstrations of OOS capabilities, with more tests planned for the near future. Furthermore, DARPA has issued an initial request for information from industry players for the design of a geosynchronous OOS project, and this request explicitly called for the implementation of OOS as a public-private partnership [27]. From here, private industry will take over the construction and operation of the infrastructure, with public support in the form of funding and regulatory oversight. The initial viability of the infrastructure will then be demonstrated on defense and government assets, followed by a string of requests from private operators to have their own spacecraft receive services. Finally, geosynchronous orbit would enter an era marked by the regular provision of services to a variety of customer spacecraft, and space architectures would benefit significantly from the launching of less massive and more technologically-agile satellites.

\section{ACKNOWLEDGEMENTS}

This work is sponsored in part by the United States Department of Defense under Contract FA8721-05C-0002. Opinions, interpretations, conclusions and recommendations are those of the authors and are not necessarily endorsed by the United States Government. 


\section{References}

[1] Union of Concerned Scientists. (2014, August 1). UCS Satellite Database. Retrieved from http://www.ucsusa.org/nuclear_weapons_and_global_security/solutions/space-weapons/ucs-satellitedatabase.html\#.VF03kSJ4pKA

[2] Saleh, J. (2008, January). Flawed Metrics: Satellite Cost per Transponder and Cost per Day. IEEE Transactions on Aerospace and Electronic Systems, 44(1), 147-156.

[3] Jones, Alan P. and Brian R. Spence (2011, August). Spacecraft solar array technology trends. IEEE Aerospace and Electronic Systems Magazine, 26(8), 17-28.

[4] Wertz, J. R., Everett, D. F., \& Puschell, J. J. (Eds.). (2011). Space Mission Engineering: The New $S M A D$. Microcosm Press.

[5] Long, A., Richards, M., \& Hastings, D. E. (2007). On-Orbit Servicing: A New Value Proposition for Satellite Design and Operation. Journal of Spacecraft and Rockets, 44(4), 964-976.

[6] Walker, J. (2007, March). Orbital Express. Fact Sheet: Defense Advanced Research Projects Agency. Arlington, VA.

[7] Orbital Express On-Orbit Mission Updates. (2007, July). Retrieved January 2015, from DARPA Tactical Technology Office: http://archive.darpa.mil/orbitalexpress/mission_updates.html

[8] Phoenix Goals. (2015). Retrieved January 2015, from DARPA Tactical Technology Office: http://www.darpa.mil/Our_Work/TTO/Programs/Phoenix/Program_Goals.aspx

[9] Escobedo Jr., V. M. (2014, October 21). Robotic Refueling Mission (RRM). Retrieved January 2015, from International Space Station Research \& Technology:

http://www.nasa.gov/mission_pages/station/research/experiments/778.html\#publications

[10] Robotic Refueling Mission. (2015, January). Retrieved from NASA Satellite Servicing Capabilities Office: http://ssco.gsfc.nasa.gov/robotic_refueling_mission.html

[11] Team Hubble: Servicing Missions. (2015). Retrieved January 2015, from Hubble Site:

http://hubblesite.org/the_telescope/team_hubble/servicing_missions.php

[12] NASA Goddard Space Flight Center. (2010). On-Orbit Satellite Servicing Study: Project Report. Greenbelt, MD.

[13] Tversky, A., \& Kahneman, D. (1974, September). Judgement under Uncertainty: Heuristics and Biases. Science, 185(4157), 1124-1131.

[14] Sterman, J. (1989, March). Modeling Managerial Behavior: Misperceptions of Feedback in a Dynamic Decisionmaking Experiment. Management Science, 35(3), 321-339.

[15] Tversky, A., \& Kahneman, D. (1979, March). Prospect Theory: An Analysis of Decision Under Risk. Econometrica, 47(2), 263-291.

[16] Trautmann, S., Vieider, F., \& Wakker, P. (2008, May). Causes of ambiguity aversion: Known versus unknown preferences. Journal of Risk and Uncertainty, 36, 225-243. 
[17] Sterman, J. (2000). Business Dynamics: Systems Thinking and Modeling for a Complex World. Boston: Irwin/McGraw-Hill.

[18] Putbrese, Benjamin L. (2015, June). Exploring Design and Policy Options for Orbital Infrastructure Projects. SM Thesis. Cambridge: MIT.

[19] Clark, S. (2015, March 1). Boeing's first two all-electric satellites ready for launch. Retrieved 2015, from Spaceflight Now: http://spaceflightnow.com/2015/03/01/boeings-first-two-all-electric-satellitesready-for-launch/

[20] Courtland, R. (2014, December 10). DARPA Prepares to Launch "Satlets". Retrieved 2015, from IEEE Spectrum: http://spectrum.ieee.org/tech-talk/aerospace/satellites/darpas-satellite-revival-programgears-up-for-first-launch

[21] Saleh, Joseph H.; Lamassoure, Elisabeth S.; Hastings, Daniel E.; \& Newman, Dava J. (2003). Flexibility and the Value of On-Orbit Servicing: New Customer-Centric Perspective. Journal of Spacecraft and Rockets, 40(2), 279-291.

[22] Richards, M. (2006). On-Orbit Serviceability of Space System Architectures. SM Thesis. Cambridge: MIT.

[23] Mai, T. (2014, October 3). Near Earth Network (NEN). Retrieved from NASA Space Communications and Navigation (SCaN):

https://www.nasa.gov/directorates/heo/scan/services/networks/txt_nen.html

[24] Belcher, D., Freese, S., Laygo, K., \& Osborn, D. (2014). Analysis of United States Policy and Legal Impediments to On-Orbit Satellite Servicing Activities. 64th International Astronautical Congress.

[25] Clore, R. E. (2012). International Developments in Global Navigation Satellite Systems. Washington, D.C.: U.S. Department of State: Office of Space and Advanced Technology.

[26] Anderson, C. (2013). Rethinking public-private space travel. Space Policy, 29(4), 266-271.

[27] DARPA. (2014, September 3). Wanted: Insights to Guide Creation of Robotic Satellite-Servicing Capabilities in Geostationary Earth Orbit. Retrieved 2015, from

http://www.darpa.mil/NewsEvents/Releases/2014/09/03.aspx

[28] (1967). Treaty on Principles Governing the Activities of States in the Exploration and Use of Outer Space, Including the Moon and Other Celestial Bodies. United Nations General Assembly. 